\section{Stereotactic and Functional Neurosurgery}

\title{
Automated 3-Dimensional Brain Atlas Fitting to Microelectrode Recordings from Deep Brain Stimulation Surgeries
}

\author{
J. Luis Luján ${ }^{a}$ Angela M. Noecker ${ }^{a} \quad$ Christopher R. Butson ${ }^{a}$ Scott E. Cooper ${ }^{b}$ \\ Benjamin L. Walter ${ }^{b}$ Jerrold L. Vitek ${ }^{b, c}$ Cameron C. Mclntyre ${ }^{a, b}$ \\ ${ }^{a}$ Department of Biomedical Engineering, ${ }^{b}$ Center for Neurological Restoration, and \\ 'Department of Neurosciences, Cleveland Clinic Foundation, Cleveland, Ohio, USA
}

\section{Key Words}

Deep brain stimulation - Brain atlas · Microelectrode

recordings $\cdot$ Stereotactic neurosurgery $\cdot$ Stereotactic target location

\begin{abstract}
Objective: Deep brain stimulation (DBS) surgeries commonly rely on brain atlases and microelectrode recordings (MER) to help identify the target location for electrode implantation. We present an automated method for optimally fitting a 3-dimensional brain atlas to intraoperative MER and predicting a target DBS electrode location in stereotactic coordinates for the patient. Methods: We retrospectively fit a 3dimensional brain atlas to MER points from 10 DBS surgeries targeting the subthalamic nucleus (STN). We used a constrained optimization algorithm to maximize the MER points correctly fitted (i.e., contained) within the appropriate atlas nuclei. We compared our optimization approach to conventional anterior commissure-posterior commissure (AC/PC) scaling, and to manual fits performed by four experts. A theoretical DBS electrode target location in the dorsal STN was customized to each patient as part of the fitting process and compared to the location of the clinically defined therapeutic stimulation contact. Results: The human expert and computer optimization fits achieved significantly better fits than
\end{abstract}

the AC/PC scaling $(80,81$, and $41 \%$ of correctly fitted MER, respectively). However, the optimization fits were performed in less time than the expert fits and converged to a single solution for each patient, eliminating interexpert variance. Conclusions and Significance: DBS therapeutic outcomes are directly related to electrode implantation accuracy. Our automated fitting techniques may aid in the surgical decision-making process by optimally integrating brain atlas and intraoperative neurophysiological data to provide a visual guide for target identification.

Copyright $\odot 2009$ S. Karger AG, Basel

\section{Introduction}

Deep brain stimulation (DBS) is a surgical intervention that uses electrical stimulation to treat the symptoms of medically refractory neurological disorders such as Parkinson's disease (PD) [1-4]. The surgical strategy for DBS varies among centers $[1,5,6]$, but generally involves the integration of multiple data types. These can include various imaging modalities, intraoperative electrophysiological mapping [i.e., $\mathrm{x}, \mathrm{y}$, and $\mathrm{z}$ microelectrode recording (MER) coordinate points] [5-9], and standardized anatomical atlases [10] to define the DBS electrode trajectory and target implantation location $[1,3]$.

\section{KARGER}

๑ 2009 S. Karger AG, Basel

Fax +41613061234 E-Mail karger@karger.ch www.karger.com www.karger.com/sfn
Cameron C. McIntyre, $\mathrm{PhD}$

Department of Biomedical Engineering, Cleveland Clinic Foundation

9500 Euclid Avenue ND20

Cleveland, OH 44195 (USA)

Tel. +1 216445 3264, Fax +1 216444 9198, E-Mail mcintyc@ccf.org 
Table 1. Fit comparison

\begin{tabular}{|c|c|c|c|c|c|c|c|c|c|c|c|c|c|c|c|}
\hline \multirow{2}{*}{$\begin{array}{l}\text { Patient } \\
\text { No. }\end{array}$} & \multicolumn{5}{|c|}{ Expert-defined } & \multicolumn{5}{|c|}{ Optimization } & \multicolumn{5}{|c|}{ AC/PC scaling } \\
\hline & $\begin{array}{l}\mathrm{C}_{\mathrm{THA}} \\
\%\end{array}$ & $\begin{array}{l}\mathrm{C}_{\mathrm{STN}} \\
\%\end{array}$ & $\begin{array}{l}\text { I } \\
\%\end{array}$ & $\begin{array}{l}\mathrm{FE} \\
\mathrm{mm}\end{array}$ & $\mathrm{t}$ min & $\begin{array}{l}\mathrm{C}_{\mathrm{THA}} \\
\%\end{array}$ & $\begin{array}{l}\mathrm{C}_{\mathrm{STN}} \\
\%\end{array}$ & $\begin{array}{l}\text { I } \\
\%\end{array}$ & $\begin{array}{l}\mathrm{FE} \\
\mathrm{mm}\end{array}$ & $\mathrm{t}$ & $\begin{array}{l}\mathrm{C}_{\mathrm{THA}} \\
\%\end{array}$ & $\begin{array}{l}\mathrm{C}_{\mathrm{STN}} \\
\%\end{array}$ & $\begin{array}{l}\text { I } \\
\%\end{array}$ & $\begin{array}{l}\mathrm{FE} \\
\mathrm{mm}\end{array}$ & $\begin{array}{l}\mathrm{t} \\
\min \end{array}$ \\
\hline 1 & 84 & 95 & 0 & 1.7 & 5.5 & 96 & 96 & 0 & 0.1 & 0.1 & 80 & 50 & 2 & 24.7 & $\mathrm{~N} / \mathrm{A}$ \\
\hline 2 & 79 & 96 & 1 & 40.0 & 8.4 & 90 & 71 & 0 & 7.5 & 1.0 & 29 & 52 & 3 & 466.2 & N/A \\
\hline 3 & 56 & 90 & 1 & 46.3 & 7.9 & 73 & 76 & 7 & 8.2 & 0.4 & 52 & 32 & 7 & 99.5 & $\mathrm{~N} / \mathrm{A}$ \\
\hline 4 & 70 & 81 & 0 & 23.0 & 10.7 & 79 & 70 & 0 & 7.0 & 0.2 & 41 & 26 & 10 & 205.0 & $\mathrm{~N} / \mathrm{A}$ \\
\hline 5 & 90 & 80 & 2 & 36.4 & 11.3 & 82 & 69 & 2 & 26.2 & 1.2 & 47 & 38 & 2 & 304.7 & $\mathrm{~N} / \mathrm{A}$ \\
\hline 6 & 71 & 89 & 1 & 71.9 & 9.4 & 79 & 69 & 6 & 19.7 & 0.3 & 51 & 51 & 3 & 161.8 & $\mathrm{~N} / \mathrm{A}$ \\
\hline 7 & 67 & 79 & 0 & 40.0 & 9.9 & 95 & 74 & 5 & 3.9 & 0.2 & 37 & 53 & 0 & 148.0 & $\mathrm{~N} / \mathrm{A}$ \\
\hline 8 & 53 & 97 & 1 & 22.0 & 8.2 & 76 & 90 & 0 & 1.8 & 0.2 & 0 & 76 & 7 & 109.4 & $\mathrm{~N} / \mathrm{A}$ \\
\hline 9 & 62 & 88 & 0 & 59.1 & 7.7 & 87 & 71 & 0 & 27.3 & 0.4 & 69 & 45 & 0 & 120.0 & N/A \\
\hline 10 & 75 & 98 & 0 & 7.9 & 6.9 & 90 & 88 & 1 & 0.9 & 0.1 & 0 & 8 & 0 & 195.4 & $\mathrm{~N} / \mathrm{A}$ \\
\hline
\end{tabular}

Percentage of thalamic (THA) and subthalamic (STN) MER points correctly (C) and incorrectly (I) fitted by the brain atlas, along with fitting error (FE) and fitting time $(\mathrm{t})$ for the expert-defined, optimization, and conventional AC/PC scaling methods.

A fundamental purpose of MER is to verify the stereotactic location of the target brain region for electrode implantation. A neurophysiologist records neural activity as a microelectrode is advanced through stereotactic brain space and identifies specific nuclei based on the properties of the neural signals recorded $[3,5-7,11-13]$. While not performed at all DBS centers, this verification process can help alleviate two limitations. First, the surgical target cannot always be directly identified on preoperative imaging data [14]. Second, the intracranial pressure changes once the burr hole is drilled, resulting in a possible shift of the subcortical structures, thereby altering the position of the target point in stereotactic space [15-17].

Brain atlases are commonly employed to assist in the definition of the initial target point and interpret the MER [10]. However, standard 2-dimensional atlas planes seldom correspond to the oblique trajectories used, which along with the limited resolution and discontinuity of the atlas slices may delay the atlas-to-MER fitting process and/or increase the number of subsequent electrode tracks required to define the optimal implantation location $[18,19]$. Thus, DBS surgical navigation systems that better integrate the various data sets used in electrode implantation are currently under development $[6,7,14$, 19, 20]. Additionally, 3-dimensional atlases have been created by interpolating between 2-dimensional slices $[14,21]$ or by generating maps that show the functional borders of target nuclei $[6,7,14,20,22]$. However, commercial surgical navigation systems do not currently provide an option to refit the brain atlas to the MER after it has been fitted to the preoperative imaging data, or entail a manual refitting, which requires precious time in the operating room and may be inconsistent across users. To address these limitations, we present a method that automatically fits a 3-dimensional brain atlas to patient-specific intraoperative MER using our academic DBS research software system, Cicerone [19].

\section{Methods}

\section{Subject Population}

Ten subthalamic nucleus (STN) DBS patients were retrospectively selected from a database of patients who had undergone surgery at the Cleveland Clinic. Unilateral implantation of a DBS electrode (Model 3387 or 3389, Medtronic Inc., Minneapolis, Minn., USA) was performed on the left side of the brain in 4 patients (patients 1, 4-6; table 1) and on the right side of the brain in 6 patients (patients 2, 3, 7-10). Each patient had idiopathic PD that responded to dopamine replacement therapy; however, the patients exhibited a wide spectrum of therapeutic DBS outcomes (see Maks et al. [23] for additional documentation on these 10 patients). This diverse patient population was selected to provide a realistic representation of the variability encountered with STN DBS for PD. This study was approved by the Cleveland Clinic Institutional Review Board.

Microelectrode Recordings

The technical aspects of MER acquisition are presented elsewhere [5]. Briefly, MER were performed with platinum-iridium microelectrodes (FHC Inc., Bowdoinham, Me., USA) that exhibited typical impedances in the range of 0.3-0.6 M $\Omega$. Electrodes were inserted using a microTargeting ${ }^{\circledR}$ microdrive (FHC Inc.) attached to a stereotactic frame (Leksell model G, Elekta Corp., 

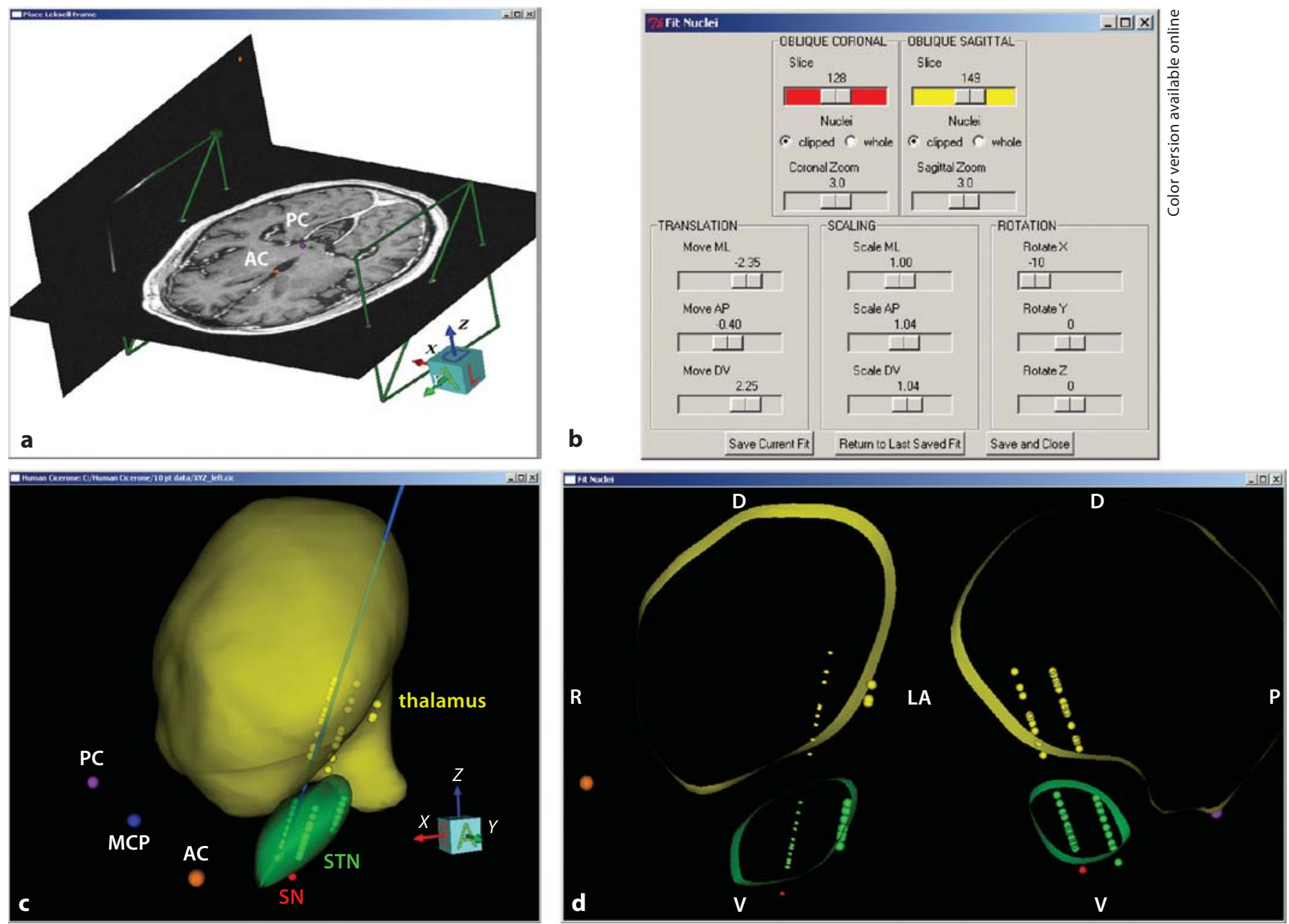

Fig. 1. Graphical user interface. a Stereotactic frame and AC/PC definition based on the patient's preoperative MRI. b-d Manual brain atlas fitting interface as seen by each of the human experts. b Control panel for manipulation of the brain atlas to fit the MER points. c Three-dimensional rendering of the atlas nuclei and all of the MER points (left side of the brain, patient 1). Thalamic MER points (yellow in the online version), STN MER points (green in

the online version), and substantia nigra (SN) MER points (red in the online version). The AC, PC, and mid-commissural point (MCP) are also shown. d Two-dimensional oblique sagittal and oblique coronal views parallel to the microelectrode trajectory for the data shown in c. Only MER points in the given plane are shown.

Stockholm, Sweden). An initial MER track was directed towards an MR image-based target. If the neural recordings in this penetration did not suggest an optimal location (e.g. less than $5 \mathrm{~mm}$ thickness, no somatosensory driving, low-threshold microstimulation effects suggesting proximity to internal capsule or central track of oculomotor nerve), then additional penetrations were required to refine the target location. This patient population received an average of $4.8 \pm 1.3$ microelectrode tracks oriented with ring angles of $62.8 \pm 6.8^{\circ}$ and arc angles of $8.5 \pm 2.6$ or 15.4 $\pm 2.8^{\circ}$ from the vertical for left and right implants, respectively. Tracks had an average of $22 \pm 12$ clinically recorded MER sites. Each MER site (fig. 1c, d) was analyzed and classified intraoperatively by one of three experienced neurophysiologists (J.L.V., S. E.C., B.L.W.) using standard criteria $[5,8]$.

Brain Atlas

The 3-dimensional brain atlas used in this study was originally developed by Surgical Navigation Technologies (Medtronic Inc.) and Jaimie Henderson (Stanford University, Palo Alto, Calif., USA). Additional details on the origin of the brain atlas can be found in the methods of Butson et al. [24]. Briefly, 3-dimensional surfaces representing visible subcortical nuclei (e.g. thalamus) were segmented from a high-resolution MRI. These surfaces were then aligned with 2-dimensional Schaltenbrand and Wahren slices [10] to define outlines of nonvisible nuclei (e.g. STN) and lofted to create the additional 3-dimensional surfaces. For the purposes of this study, we used atlas representations of the thalamus and STN (fig. 2). 


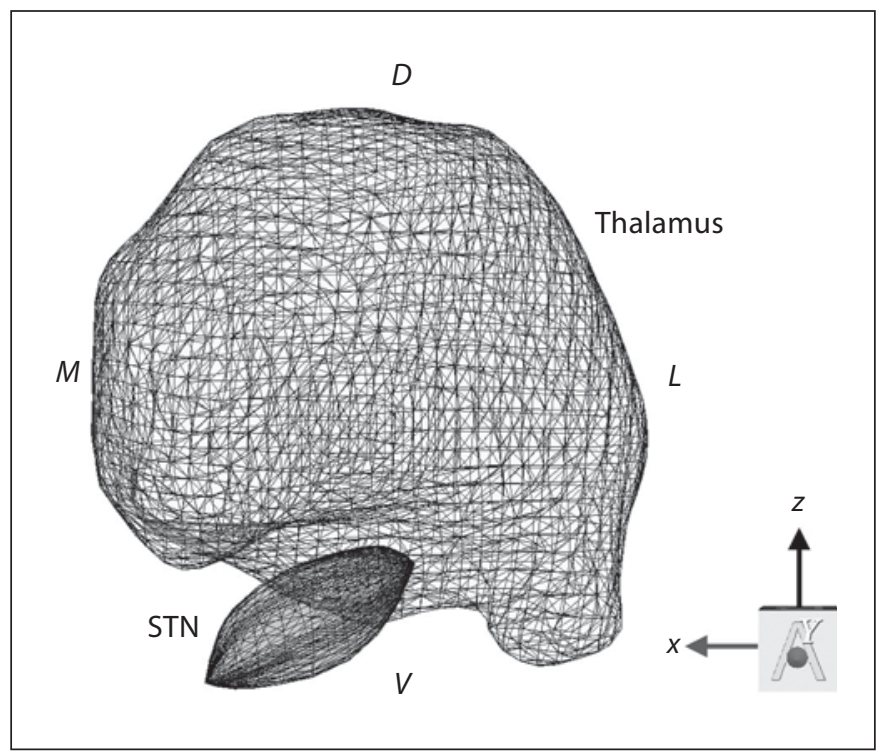

Fig. 2. The brain atlas is a collection of nuclei, where each nucleus is formed by a 3-dimensional polygonal surface (wireframe). Anterior view of the left thalamus (light gray) and STN (dark gray) is shown.

\section{Traditional Brain Atlas Fits}

We fit the brain atlas to each patient using anterior commissure-posterior commissure (AC/PC) scaling and human expertdefined fits. Each patient data set (imaging data and MER points) was registered into stereotactic space using fiducial markers [19] to obtain an explicit definition of the coordinates of the AC, PC, and each MER site in a rigid coordinate system (fig. 1). For the $\mathrm{AC} / \mathrm{PC}$ scaling, the brain atlas was seeded into stereotactic space by orienting it to the midline and scaling it along the anteroposterior axis such that the atlas $\mathrm{AC} / \mathrm{PC}$ line matched that of each patient. Manual fits of the 3-dimensional brain atlas to the MER points were performed by four experienced human experts (J.L.V., S.E.C., B.L.W., C.C.M.). A custom software application (derived from Cicerone [19]) was developed to enable the human users to interactively fit the brain atlas to the MER points. This application presented the expert users with three interactive views of the MER points and brain atlas. The first view was a 3-dimensional rendering of the atlas nuclei and all of the MER points (fig. 1c). The second and third views, respectively, were 2-dimensional 1$\mathrm{mm}$-thick oblique sagittal and oblique coronal views parallel to the actual microelectrode trajectory (fig. 1d). These views showed only MER points on that oblique plane. The users could manipulate the fit of the brain atlas to the MER points via a control panel (fig. 1b), and could interactively move through different slices in various planes to view the data in a more traditional 2-dimensional format. The viewing windows interactively showed the transformed brain atlas as the user translated, scaled, and rotated the brain atlas ( 9 degrees of freedom). The human experts spent time familiarizing themselves with the controls of the software using data from a practice patient prior to this study. Once the users were comfortable with the task, they were allowed a maxi- mum of 15 min to perform the fit of each patient using the same transformation ranges available to the optimization algorithm.

\section{Optimized Brain Atlas Fit}

The goal of the optimization algorithm was to define a set of linear transformations that maximized the number of neurophysiologically identified MER sites inside their respective atlasdefined nuclei (i.e., thalamus and STN) and minimized inclusion of MER points from other areas of the brain (e.g. substantia nigra, zona incerta). Additionally, the optimization algorithm minimized the overall euclidean distance between the surfaces of the atlas nuclei and their corresponding MER points not contained by the atlas. The original anatomical relationships between atlas nuclei were maintained throughout the fit process (i.e., nuclei were scaled, translated and rotated together, not independently).

The optimal location of the brain atlas with respect to the MER was found by minimizing a cost function (see Appendix) that depended on the current set of bounded linear transformations [25]. The cost function was defined by two terms: (1) the weighted sum of the squared minimum euclidean distances between neurophysiologically identified MER points and the surfaces of their corresponding nuclei (fig. 3), and (2) the weighted sum of MER points incorrectly fit by the atlas. The MER-to-atlas euclidean distances were defined as zero when MER points were contained within their corresponding nuclei. The terms in the cost function were weighted according to their specific nucleus. Larger weights for the STN $\left(\mathrm{W}_{\mathrm{STN}}=20\right)$ were used to assign a higher priority to fitting the STN over other nuclei $\left(\mathrm{W}_{\mathrm{THA}}=2\right)$. The cost function was calculated for different sets of transformations obtained by the optimization algorithm over a transformation space defined by a maximum rotation of $\pm 10^{\circ}$, a maximum translation of $\pm 10 \mathrm{~mm}$, and a maximum scaling of $\pm 20 \%$ for each axis from the initial condition. These bounds allow for a large degree of interpatient variability without creating an excessively large search space for the solution.

\section{DBS Target Electrode Location}

We determined a target location for the active DBS electrode contact with respect to the atlas STN. This target, selected only as an illustrative example, was defined $2 \mathrm{~mm}$ dorsal to the geometric centroid of the STN in the original brain atlas geometry (fig. 4). This region has been previously identified as a general target location for therapeutic DBS in PD [12, 23, 26-28], and could be suggested as an ideal location to place contact 2 of the clinical DBS electrode.

The stereotactic location of this theoretical target point was transformed for each patient as the brain atlas was fit to their MER data. We then compared the location of the transformed target point to the stereotactic coordinates of the monopolar DBS electrode contact used for therapeutic stimulation in each patient (i.e., active contact). The therapeutic contact was determined by traditional postoperative clinical programming and the actual stereotactic location of the DBS electrode was confirmed by coregistration of the postoperative CT into stereotactic space prior to this study [23].

\section{Fit Evaluation}

We evaluated each of the three fitting techniques (AC/PC scaling, human expert, and optimization algorithm) by comparing five metrics: (1) the percentage of MER points correctly contained 


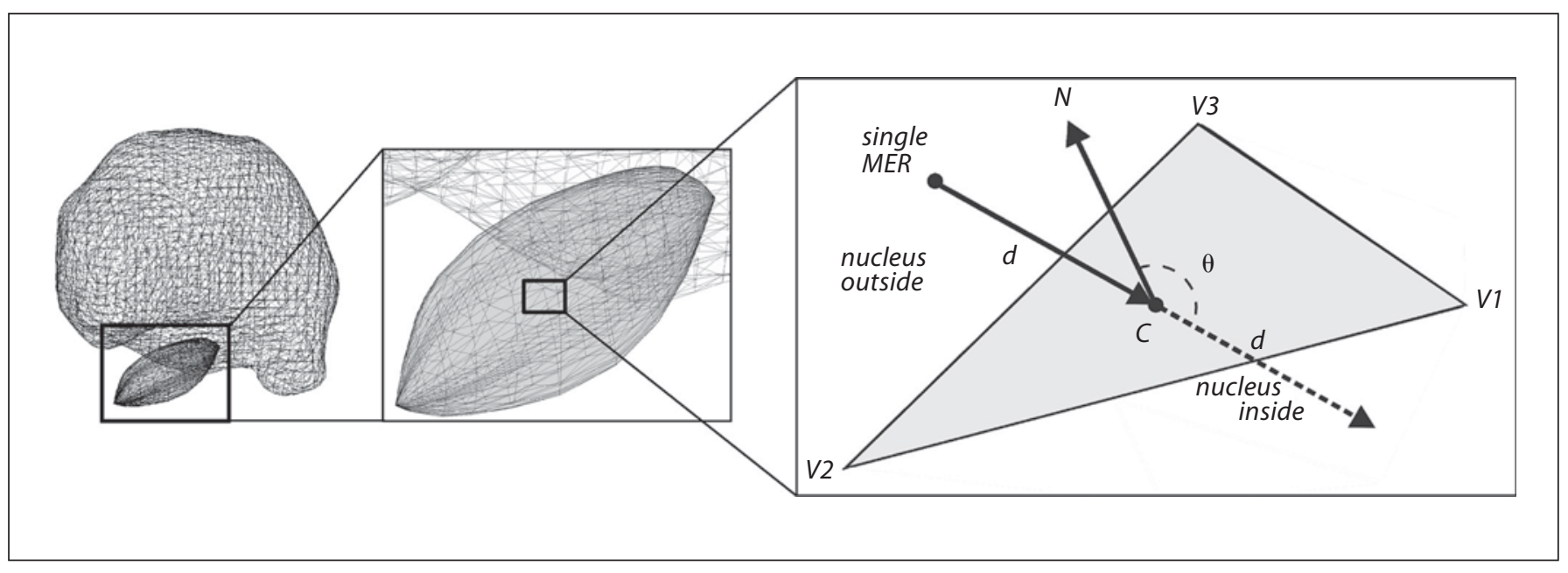

Fig. 3. Schematic representation of an STN MER site and its closest polygon on the surface of the atlas STN. The plane of the polygon given by the three vertices ( V1, V2, and V3) separates the inside and outside of the nucleus with respect to the MER site. In this example, the MER site is outside the nucleus because the angle between the vector normal $(N)$ to the surface polygon, and the distance vector $(d)$ defined by the MER site and the polygon centroid $(C)$ is greater than $90^{\circ}$. (i.e., fit) within their respective nuclei, (2) the percentage of MER points incorrectly contained within any nuclei, (3) the summed distance of unfitted MER points to their corresponding nuclei surfaces (fitting error), (4) the fitting time, and (5) euclidean distance from the active DBS electrode contact to the atlas-defined target electrode location. The percentage of MER points correctly and incorrectly contained by the atlas quantified the goodness of fit for each method. The fitting error discriminated between fits for which the atlases contained the same percentage of MER points but for which different transformations were used. We performed one-way ANOVA using Origin 7.5 (OriginLab Corporation, Northampton, Mass., USA) to determine statistical significance.

\section{Sensitivity Analysis}

We performed a sensitivity analysis to address the effects of changing the STN weights from 1 to 20 times the weight associated with the thalamus. Additionally, we evaluated the ability of the brain atlas fit to accommodate all of the MER points acquired in a patient, while incrementally adding individual tracks to the optimization algorithm. This evaluation was performed from one up to the maximum number of tracks recorded for each patient (i.e., up to six tracks) in the order in which these tracks were acquired clinically.

\section{Results}

The fitting error (i.e., atlas-to-MER summed distance) was significantly smaller for the optimization method (10 $\pm 10 \mathrm{~mm})$ than for the human expert $(35 \pm 22 \mathrm{~mm}, \mathrm{p}<$ $0.005)$ and $\mathrm{AC} / \mathrm{PC}$ scaling $(183 \pm 124 \mathrm{~mm}, \mathrm{p}<0.001)$

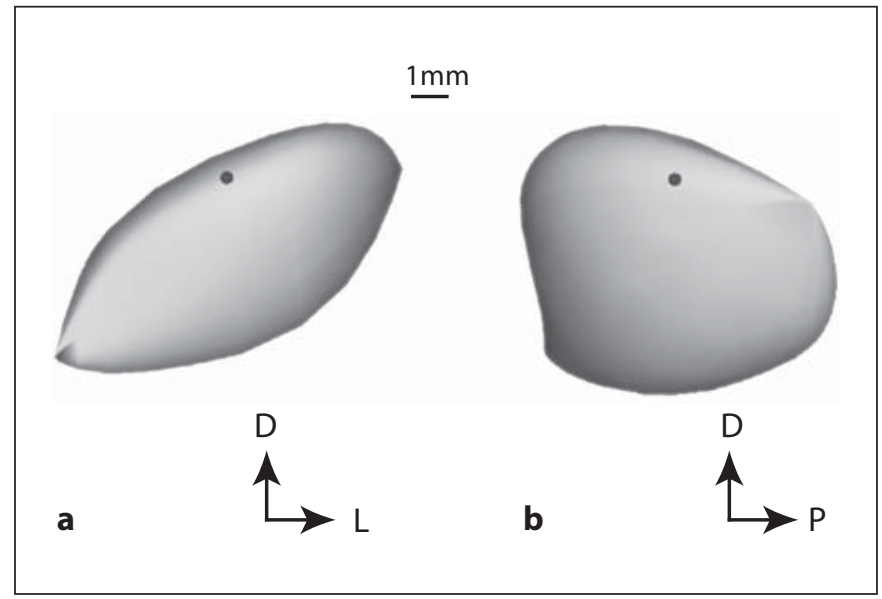

Fig. 4. Theoretical DBS electrode target (dark dot) in the dorsal STN. a Coronal view. b Sagittal view. The arrows show the dorsolateral (D-L) and dorsoposterior (D-P) directions.

methods (fig. 5d). The human expert and optimization fits resulted in a brain atlas that correctly fit an average of $80 \pm 10$ and $81 \pm 9 \%$ of the MER points, respectively (fig. 5b), while AC/PC scaling only fit $42 \pm 22 \%$ (table 1 ). The percentage of MER points correctly fit by $\mathrm{AC} / \mathrm{PC}$ scaling was significantly smaller $(p<0.001)$ than the human expert and optimization fits. The correctly fit MER points for the optimized and human expert fits were not 


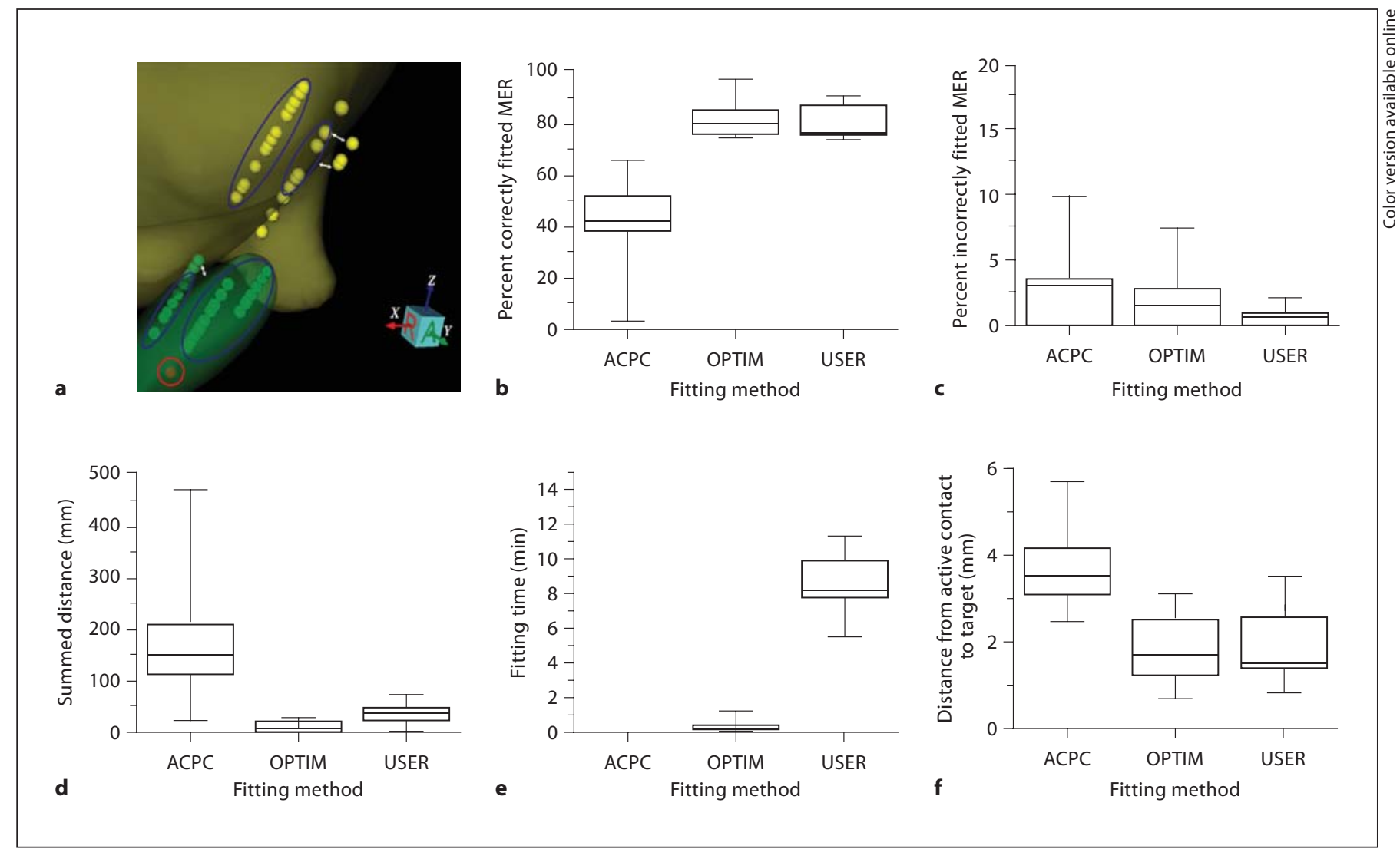

Fig. 5. Fit comparison. a Visualization of the metrics used to determine goodness of fit. The ovals show thalamic and STN MER points correctly fit by the atlas. The circle shows an MER point from the substantia nigra that was incorrectly fitted in the atlas STN. The white arrows represent the euclidean distance from MER points not fitted by the atlas to the closest polygonal centroid on the surface of their corresponding atlas nucleus. b Percentage of MER points correctly fitted by the atlas. c Percentage of MER points incorrectly fitted by the atlas. $\mathbf{d}$ Summed distance (fitting error). e Fitting time (AC/PC scaling was the start point for our analysis and thus not timed). $f$ Distance from the clinically defined active electrode contact to the theoretical target location after the atlas was fitted with each method. b-f The boxes contain the middle $50 \%$ of the data for each metric. The upper and lower edges of the boxes indicate the 75 th and 25 th percentile of the corresponding data set while the line in the box indicates the median value of the data. The ends of the vertical lines indicate the minimum and maximum data values for each metric. statistically different $(\mathrm{p}=0.7)$. The human expert and optimization fits differed only in the percentages of MER points incorrectly fit (fig. 5c). The percentages of MER points incorrectly fit by the human expert (0.5\%) fittings were significantly smaller than those of the optimization $(2.2 \%, \mathrm{p}<0.04)$ and $\mathrm{AC} / \mathrm{PC}$ fittings $(3.5 \%, \mathrm{p}<0.005)$. There were no significant differences between the optimized and AC/PC fittings with respect to incorrectly fit MER points $(\mathrm{p}=0.3)$.

The human expert and optimization fits were effectively equivalent from a qualitative perspective (i.e., both fits captured the electrophysiology of the patients in a comparable way). However, the optimization fits were achieved in $0.4 \pm 0.4 \mathrm{~min}$ on a $1.32-\mathrm{GHz}$ Lenovo laptop computer with 2 GB of RAM (Lenovo, Morrisville, N.C., USA) running Windows XP (Microsoft Co., Redmond, Wash., USA), while the human experts used $8.6 \pm 1.8$ min to achieve their fits (fig. 5e). The optimization algorithm always generated the same fit for a specific data set (i.e., MER points), indicating a unique solution given the parameters of the algorithm. In contrast, the human expert fits exhibited a noticeable degree of variability in their fits for the same data (fig. 6).

The average distance from the atlas-based target to the location of the active electrode contact for the human expert, optimization, and AC/PC fitting methods were 1.9 

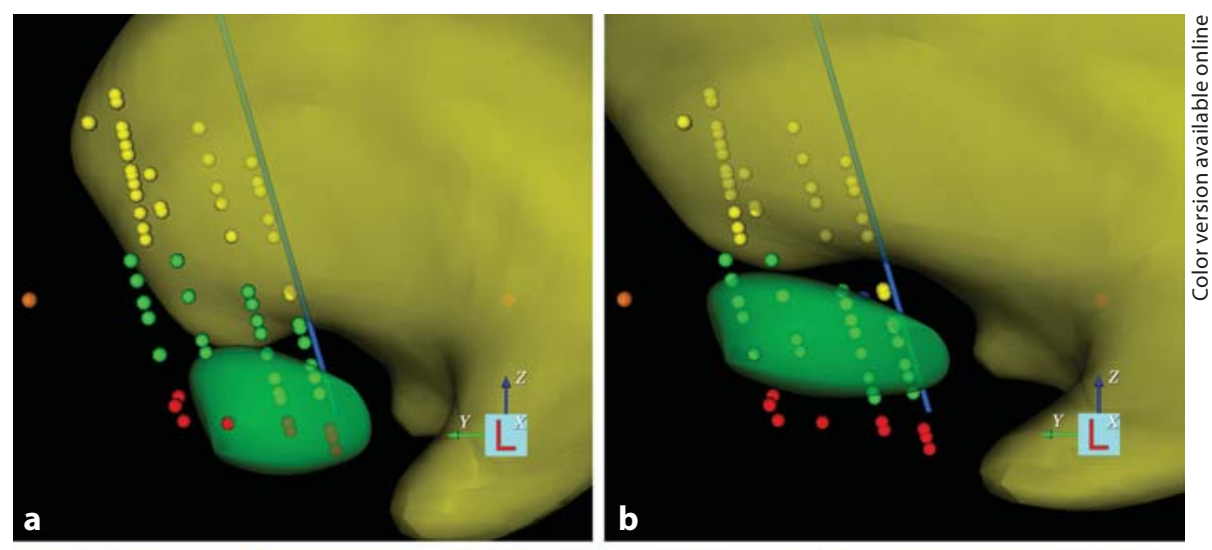

Fig. 6. Fit comparison. Oblique sagittal views of a single patient's left side atlas fittings to the MER points for the AC/PC scaling method (a), optimization-based fitting (b), and expert-defined fittings (c-f). The atlas-defined volumes of the thalamus and STN are shown in light grey (yellow) and grey (green), respectively (see online version). Electrophysiologically identified MER points in the thalamus and STN are shown using the colors of their corresponding nuclei. Substantia nigra MER points are shown in dark grey (red, see online version).
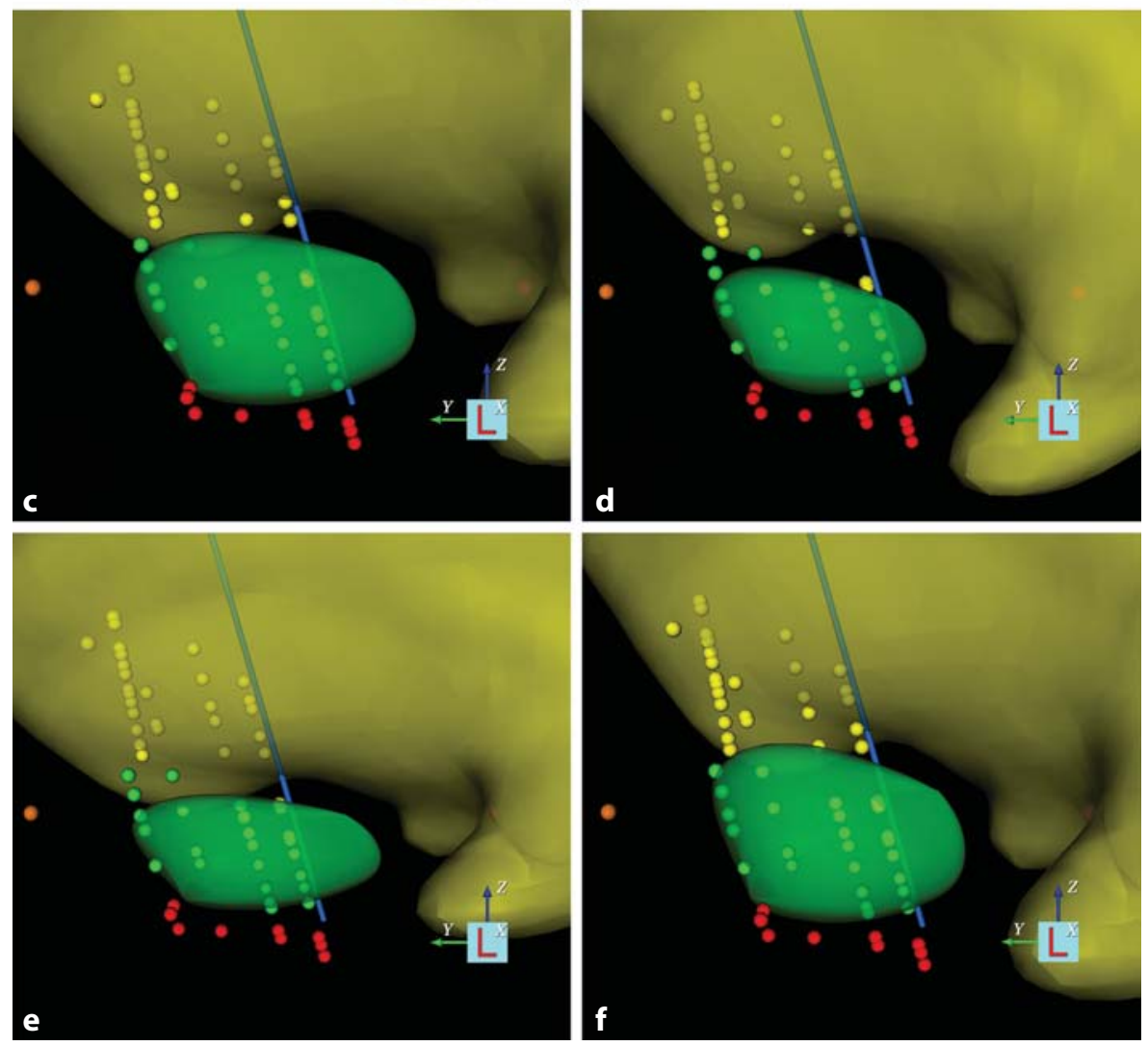

$\pm 0.8,1.9 \pm 0.7$, and $3.8 \pm 0.9 \mathrm{~mm}$, respectively (table 2 fig. 5f). The distance from the target with the AC/PC fit to the clinically defined therapeutic contact was significantly larger $(\mathrm{p}<0.01)$ than the distance generated by the other two fitting methods.

Increasing the optimization weight associated with fitting STN MER points in the STN increased the percentage of correctly fit STN MER by an average of $8 \%$ while decreasing the percentage of correctly fit thalamic MER by 7\%. However, STN weights larger than 10 times the thalamus weights did not improve the percentage of correctly fit STN MER any further (i.e., it reached a plateau). The percentages of incorrectly fit MER, and the overall summed distance were not significantly affected by the weights across the ranges evaluated $(\mathrm{p}>0.05)$.

The optimization algorithm required a minimum of two tracks of useful MER information to provide an appropriate fit (e.g. more than $61 \%$ of all MER points acquired in the patient were correctly fit with less than 


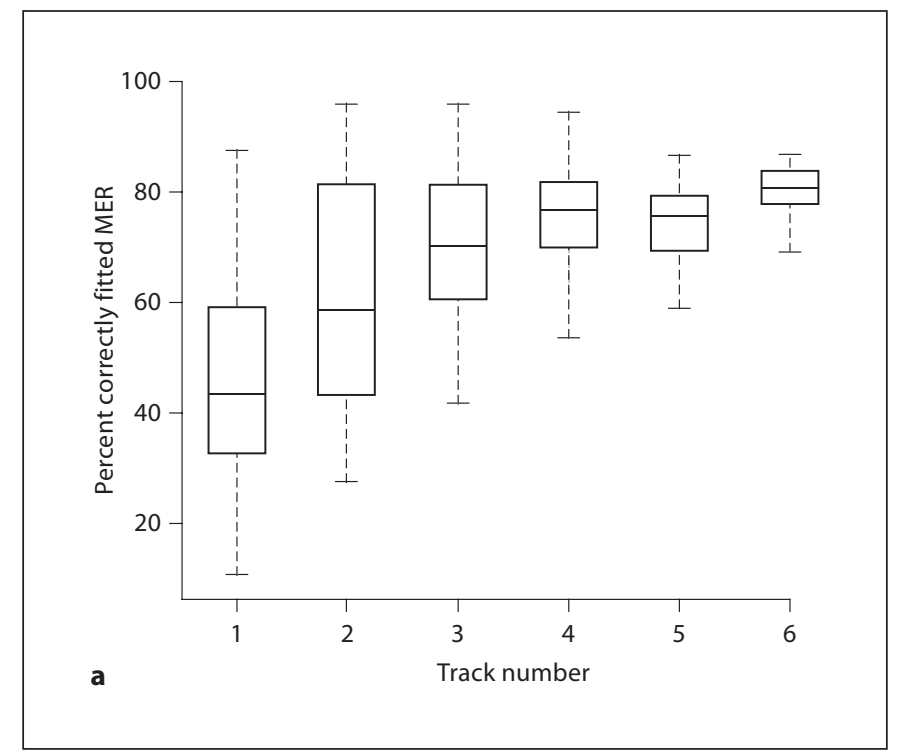

Fig. 7. Influence of the number of tracks on the fit. The optimization algorithm was incrementally given MER points from tracks in the order they were collected in the operating room. After the addition of each track, the brain atlas fit to all of the MER points collected for that patient was evaluated. a Percentage of all STN and thalamic MER points contained within their respective nuclei. b Summed euclidean distance from all STN and thalamic points not in their respective nuclei. c Displacement distance from each incremental target location prediction to the target location defined by the fit with all tracks.

$1.8 \%$ of MER incorrectly fit). With three tracks, the mean percentage of MER fit correctly increased to over $70 \%$ without increasing the percentage of incorrectly fit MER (1.5\%). Although the fits obtained with our optimization algorithm depended on the number of microelectrode tracks, incorporating more than three MER tracks did not have a significant effect in the percentages of MER correctly/incorrectly fit ( $p>0.1$ ), or the summed distance of MER points outside of their respective nuclei (fig. 7).
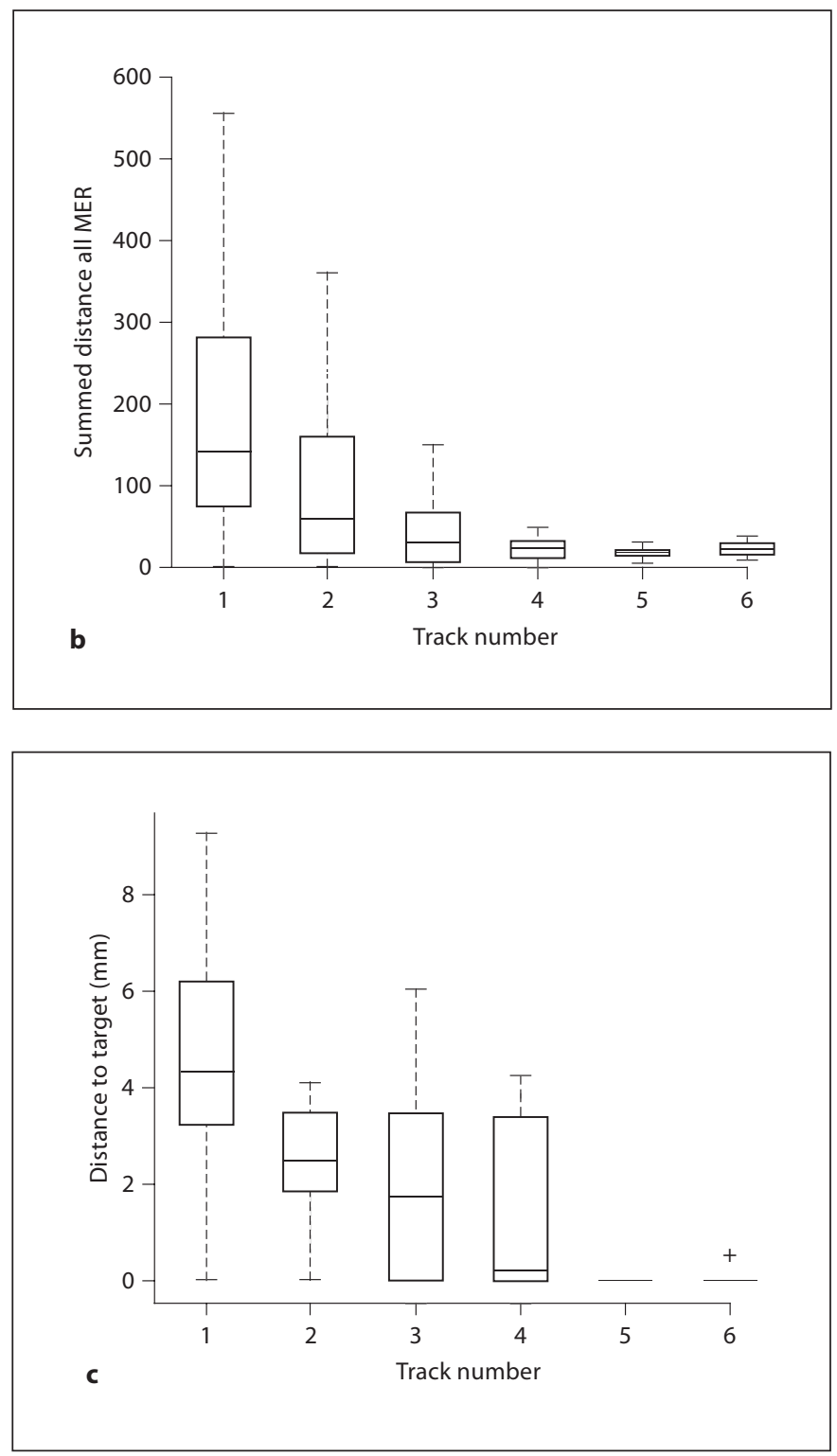

\section{Discussion}

Precise placement of DBS electrodes can facilitate maximal therapeutic outcomes with minimal side effects $[13,29]$. As such, DBS surgical targeting continues to evolve and become more precise with new advances in imaging, neurophysiology, and brain atlas technology. This proof of principle study presents a technique to automate the alignment of a generic 3-dimensional brain atlas to intraoperative MER, and thereby assist in the definition of a stereotactic target location for implantation of the DBS electrode. 
Table 2. Geometric distance between the therapeutic stimulation contact and the model-predicted location of the active therapeutic contact for the expert-defined, optimized, and conventional AC/ PC methods

\begin{tabular}{llll}
\hline $\begin{array}{l}\text { Patient } \\
\text { No. }\end{array}$ & $\begin{array}{l}\text { Expert-defined } \\
\text { model, mm }\end{array}$ & $\begin{array}{l}\text { Optimization } \\
\text { model, mm }\end{array}$ & $\begin{array}{l}\text { AC/PC scaling } \\
\text { model, mm }\end{array}$ \\
\hline 1 & 0.8 & 1.7 & 3.8 \\
2 & 1.7 & 2.7 & 4.0 \\
3 & 1.4 & 1.1 & 4.7 \\
4 & 3.5 & 3.1 & 3.0 \\
5 & 1.2 & 1.5 & 3.5 \\
6 & 2.9 & 2.1 & 3.2 \\
7 & 2.0 & 0.7 & 2.5 \\
8 & 1.5 & 2.4 & 4.2 \\
9 & 2.6 & 2.5 & 3.1 \\
10 & 1.5 & 1.2 & 5.7 \\
\hline
\end{tabular}

Recent advances in MRI make it possible to directly define target locations by direct visualization of nuclei such as the STN [30]. However, contrast and resolution limitations in mainstream MR scanners deter many from only using imaging data to make DBS implantation decisions $[6,7,12,14]$. As such, current techniques for electrode placement typically require indirect probabilistic targeting $[1,14,22,31,32]$. Furthermore, the large anatomical variability across patients provides motivation for the use of additional techniques that characterize the topography within the nucleus [5] and refine the implant location for the DBS electrode $[3,15]$.

One traditional technique for integrating the multiple data sets used in DBS surgery is the manual overlay of 2dimensional brain atlas slices that approximate specific regions of the brain onto graphs of MER points [5, 31, 33]. However, this process can be taxing given that the patient's MR images, brain atlas, and MER points are typically not coplanar, or are not available in a common visualization platform. Thus, strict adherence to the stereotactic coordinate system is typically lost, thereby limiting accuracy of the data analysis and target predictions. This study provides an example of how multiple data sets can be used together within the same stereotactic coordinate system.

Our results showed significantly larger distances between therapeutic electrode contacts and theoretical targets predicted by the conventional AC/PC method compared to the human expert and optimization fitting methods. Previous studies have proposed that there are no additional benefits from using sophisticated computer systems and high-field MRI for DBS target identification when compared to traditional indirect AC/PC-based methods [34]. In contrast, our results suggest that AC/ PC-based target identification does not adequately account for the substantial variability in patient brain geometry [30].

In general, brain atlases are associated with numerous limitations. Commonly used atlases are constructed from a single brain that may not be representative of the broader patient population or disease state. In addition, even after an atlas is fit to a patient's brain the atlas may be misleading to the true anatomy of the patient. We restricted our atlas fits to the use of linear transformations (i.e., atlas is nondeformable) to maintain the original anatomical relationships between brain nuclei. One advantage of this method is that it limits the degrees of freedom and can be used with any 3 -dimensional brain atlas. However, advances in nonlinear brain atlas deformation algorithms could increase correctly fitted MER percentages, and provide better adherence to the neuroanatomy visible in the MRI $[14,35]$. It might also be useful to include additional constraints from image-based landmarks and/or weighting schemes to account for known locations of side effects identified by macro/ microstimulation to further improve the optimizationbased fit [3].

Our optimization algorithm performed as well as experienced human experts, but in less time and without interexpert variability. Although the human expert fits achieved similar percentages of correctly fitted MER, the transformations required to position the brain atlas with respect to each patient's MER were significantly different $(p<0.05)$, particularly in three degrees of freedom: dorsoventral translation (tz), rotation about the dorsoventral (vertical) axis, and scaling along the medial-lateral direction. Thus, while the strategies followed by individual experts may provide a similar overall outcome, small differences in the fitting process can add variance to the analysis and determination of a target location. Larger fitting error variance in the human expert fit $\left(\sigma^{2}=475\right.$ $\left.\mathrm{mm}^{2}\right)$ than in the optimization fit $\left(\sigma^{2}=106 \mathrm{~mm}^{2}\right)$ suggests that the optimization algorithm provides more consistent fits.

Algorithms, similar to that presented in this study, could be developed to suggest stereotactic trajectories for MER that maximize the probability of gathering information to constrain the fit. Such an advance could reduce the number of penetrations required for target identification. For example, after a single MER track the fit between a brain atlas and the MER points is relatively un- 
constrained (fig. 7); however, acquisition of data along specific trajectories can substantially limit the possible permutations of the brain atlas to fit the data. Computational assistance in subsequent track selection could offer a degree of standardization that might benefit less experienced users by improving consistency across procedures and maximizing the a mount of information gained by each track.

Human interpretation remains the gold standard for neurophysiological identification of MER points and nuclei borders. However, the fitting performance and speed of the optimization algorithm create a realistic opportunity for its clinical use during DBS surgeries. The basic goal would be to assist human experts in their decisionmaking process. Although traditional (manual) atlas fitting techniques can be performed while the microelectrode is being retracted and readied for the next track, fast automated atlas fits (which can be updated as each new MER point is acquired) allow the surgical team to dedicate more time to critical decision-making instead of manual atlas fits.

The results of this study support the concept that computational refinement of DBS target locations based on 3-dimensional brain atlases and MER can assist electrode placement. The atlas fitting and targeting method presented here, coupled with advanced DBS surgical navigation software tools $[6,7,19,20,27]$, should improve the management of data and allow for selection of an optimal DBS electrode location in a reduced amount of time. As DBS applications and target sites continue to expand, 3dimensional renderings of atlases and trajectories may improve opportunities to optimize the stimulation of targeted brain regions with novel electrode placement strategies.

\section{Appendix}

The 3-dimensional brain atlas (fig. 2) was linearly transformed to fit the patient-specific MER (fig. 1) using an optimal set of linear transformations. These linear transformations were defined with the set of rotations $\alpha, \beta$, and $\gamma$ (about the $x, y$, and $z$ axes, respectively), translations $\left(t_{x}, t_{y}\right.$ and $\left.t_{z}\right)$, and scaling $\left(s_{x}, s_{y}\right.$, and $s_{z}$ ) of the three independent axes (fig. 8) with respect to the mid-commissural point (MCP in fig. 1c). The 3-dimensional transformations were applied to the brain atlas as a series of matrix multiplications of the form $A^{\prime}=S T A$, where $A$ and $A^{\prime}$ are $4 x n$ matrices (containing the affine coordinates of $\mathrm{n}$ vertices forming the atlas surfaces) representing the original and transformed atlases, respectively; $T$ represents the affine transformation matrix that includes rotations (applied sequentially about the $y, x$, and $z$ axes) and translations, and $S$ represents the scaling matrix transform.

$T=\left[\begin{array}{cccc}\cos \beta \cos \gamma- & -\cos \beta \sin \gamma+ & \sin \beta \cos \alpha & t_{x} \\ \sin \beta \sin \alpha \sin \gamma & \sin \beta \sin \alpha \cos \gamma & & \\ \cos \alpha \sin \gamma & \cos \alpha \cos \gamma & -\sin \alpha & t_{y} \\ -\sin \beta \cos \gamma+ & \sin \beta \sin \gamma+ & \cos \beta \cos \alpha & t_{z} \\ \cos \beta \sin \alpha \sin \gamma & \cos \beta \sin \alpha \cos \gamma & & \\ 0 & 0 & 0 & 1\end{array}\right]$

$$
S=\left[\begin{array}{cccc}
s_{x} & 0 & 0 & 0 \\
0 & s_{y} & 0 & 0 \\
0 & 0 & s_{z} & 0 \\
0 & 0 & 0 & 1
\end{array}\right]
$$

The optimal set of linear transformations was obtained by minimizing a cost function (eq. 1). The first term inside the sum calculates the weighted squared sum of the minimum euclidean distances (eq. 2) between the neurophysiologically identified MER points and the surface of their corresponding atlas nuclei. The second term calculates and weights the number of MER incorrectly fit by any nuclei (eq. 3).

$$
\begin{aligned}
& f(u)=\sum_{i}\left(W_{i} g_{i}(u)^{2}+V_{i} h_{i}(u)\right), \\
& g_{i}(u)=\sum_{j} d I_{i j}, \\
& h_{i}(u)=\sum_{l} L_{l j},
\end{aligned}
$$

where $u$ corresponds to the set of transformations, which allowed a maximum translation and rotation of $10 \mathrm{~mm}$ and $10^{\circ}$, respectively, in each direction, and a maximum scaling of $\pm 20 \%$ along each axis; $i$ and $l$ are nuclei indices describing the nucleus type (e.g. STN $=1$, thalamus $=2$ ), and $j$ is a unique index to each MER point; $W$ and $V$ are weights that allow us to prioritize the fit of different nuclei; $I_{i j}$ and $L_{l j}$ are boolean operators used to penalize the cost function for missed MER points (i.e., MER point outside its corresponding nucleus) and MER fitted within an incorrect nucleus. For this study, the STN weights $\left(W_{1}, V_{1}\right)$ were set to 20 and the weights of the thalamus $\left(W_{2}, V_{2}\right)$ were set to 2 .

To estimate the cost function for each transformation set, we first determined if the MER points were contained within their corresponding atlas nuclei. To do so, we found the polygon on the nucleus surface with the smallest euclidean distance (eq. 4) between the centroid of the polygon and its corresponding (i.e., of the same type) MER points (fig. 8). We then calculated, for this polygon, the angle (eq. 5) between the vector formed from the MER point to the centroid of the polygon (eq. 4) and the vector normal to the polygon.

$$
\begin{aligned}
& d=\sqrt{\left(M E R_{j}-C_{i j}\right)^{2}} \\
& \theta=\cos ^{-1}\left(\frac{d^{2}}{\left\|d^{2}\right\|} \cdot \frac{N_{i j}}{\left\|N_{i j}\right\|}\right)
\end{aligned}
$$




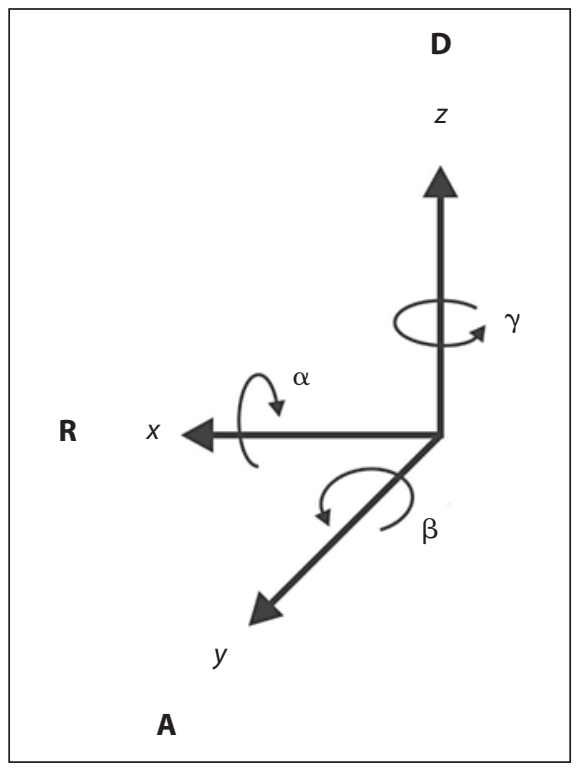

Fig. 8. $x, y$, and $z$ axes with the mid-commissural point as the origin. Also shown are the corresponding anterior-posterior (A$\mathrm{P}$ ), left-right (L-R), and dorso-ventral (D-V) directions. $\alpha, \beta$, and $\gamma$ are the angles of rotation for the $x, y$, and $z$ axes, respectively.

If the angle between these two vectors was smaller than or equal to $90^{\circ}$, the MER was contained within the nucleus and did not contribute to the fit error (i.e., atlas-to-MER summed distance) (eq. 2; by setting $I_{i j}=0$ ). On the other hand, if this angle (eq. 5) was larger than $90^{\circ}$, the MER was outside its nucleus and its distance to the nucleus surface (eq. 4) was added to the cost function (setting $I_{i j}=1$ ).
Alternatively, atlas transformations that resulted in nuclei containing MER points of a type other than their own (i.e., STN MER points contained within the thalamus) resulted in penalties to the cost function. This time, the cost function for this atlas transformation was increased by a factor of $V_{i}$ (by setting $L_{l j}=1$ ) if the MER was contained within nucleus (i.e., angle $\leq 90^{\circ}$ ). The cost function was not penalized $\left(L_{l j}=0\right)$ if the MER was outside the nucleus (i.e., angle $>90^{\circ}$ ).

The optimization algorithm was implemented using the Matlab ${ }^{\circledR}$ Optimization Toolbox's fmincon function (The MathWorks Inc., Natick, Mass., USA), which finds a local minimum of a constrained nonlinear multivariable function using sequential quadratic programming. Details on sequential quadratic programming can be found in the literature [36-39]. The optimization was initialized by using the conventional AC/PC fit for each patient. Optimization was performed for a maximum of 1,000 iterations and 3,500 function evaluations, with a constraint tolerance of 0.1 , an objective function optimality tolerance of 0.1 and a transformation parameter tolerance of 0.01 . The parameter search direction (given by the second derivative of the cost function, also known as hessian [40]) was updated according to the steepest descent method [25]. All other optimization parameters were set to their default fmincon values.

\section{Acknowledgements}

This work was supported by grants from the National Institutes of Health (R21 NS050449, R01 NS059736) and the W.H. Coulter Foundation. The authors thank Jaimie Henderson for providing the 3-dimensional atlas surfaces used in this study. J.L.L., C.R.B., and C.C.M. authored intellectual property related to this project. C.C.M. and C.R.B. hold company shares in IntElect Medical Inc. C.C.M., C.R.B., J.L.L. and A.M.N. are paid consultants of IntElect Medical Inc.

\section{References}

1 Machado A, Rezai AR, Kopell BH, Gross RE, Sharan AD, Benabid AL: Deep brain stimulation for Parkinson's disease: surgical technique and perioperative management. Mov Disord 2006;21(suppl 14):S247-S258.

2 Richter EO, Lozano AM: Deep brain stimulation for Parkinson's disease and movement disorders; in Rosch PJ (ed): Bioelectromagnetic Medicine. New York, Dekker, 2004, pp 265-276.

3 Starr PA, Christine CW, Theodosopoulos PV, Lindsey N, Byrd D, Mosley A, Marks WJ Jr: Implantation of deep brain stimulators into the subthalamic nucleus: technical approach and magnetic resonance imagingverified lead locations. J Neurosurg 2002;97: 370-387.
4 Tasker RR, Munz M, Junn FS, Kiss ZH, Davis K, Dostrovsky JO, Lozano AM: Deep brain stimulation and thalamotomy for tremor compared. Acta Neurochir 1997;68(suppl): 49-53.

5 Gross RE, Krack P, Rodriguez-Oroz MC, Rezai AR, Benabid AL: Electrophysiological mapping for the implantation of deep brain stimulators for Parkinson's disease and tremor. Mov Disord 2006;21(suppl 14):S259S283.

-6 D'Haese PF, Cetinkaya E, Konrad PE, Kao C, Dawant BM: Computer-aided placement of deep brain stimulators: from planning to intraoperative guidance. IEEE Trans Med Imaging 2005;24:1469-1478.

7 Finnis KW, Starreveld YP, Parrent AG, Sadikot AF, Peters TM: Three-dimensional database of subcortical electrophysiology for image-guided stereotactic functional neurosurgery. IEEE Trans Med Imaging 2003;22: 93-104.
8 Mogilner AY, Benabid AL, Rezai AR: Chronic therapeutic brain stimulation: history, current clinical indications, and future prospects; in Rosch PJ (ed): Bioelectromagnetic Medicine. New York, Dekker, 2004, pp 133151.

9 Andrade-Souza YM, Schwalb JM, Hamani C, Eltahawy H, Hoque T, Saint-Cyr J, Lozano AM: Comparison of three methods of targeting the subthalamic nucleus for chronic stimulation in Parkinson's disease. Neurosurgery 2005;56:360-368.

10 Schaltenbrand G, Wahren W: Atlas for Stereotaxy of the Human Brain, ed 2. Chicago, Year Book Medical Publishers, 1977.

$\checkmark 11$ Falkenberg JH, McNames J, Favre J, Burchiel $\mathrm{KJ}$ : Automatic analysis and visualization of microelectrode recording trajectories to the subthalamic nucleus: preliminary results. Stereotact Funct Neurosurg 2006;84:35-45. 
12 Littlechild P, Varma TR, Eldridge PR, Fox S, Forster A, Fletcher N, Steiger M, Byrne P, Tyler K, Flintham S: Variability in position of the subthalamic nucleus targeted by magnetic resonance imaging and microelectrode recordings as compared to atlas co-ordinates. Stereotact Funct Neurosurg 2003;80: 82-87.

13 Moran A, Bar-Gad I, Bergman H, Israel Z: Real-time refinement of subthalamic nucleus targeting using Bayesian decision-making on the root mean square measure. Mov Disord 2006;21:1425-1431.

14 Yelnik J, Bardinet E, Dormont D, Malandain G, Ourselin S, Tande D, Karachi C, Ayache N, Cornu P, Agid Y: A three-dimensional, histological and deformable atlas of the human basal ganglia. 1. Atlas construction based on immunohistochemical and MRI data. Neuroimage 2007;34:618-638.

- 15 Halpern CH, Danish SF, Baltuch GH, Jaggi JL: Brain shift during deep brain stimulation surgery for Parkinson's disease. Stereotact Funct Neurosurg 2008;86:37-43.

16 Khan MF, Mewes K, Gross RE, Skrinjar O: Assessment of brain shift related to deep brain stimulation surgery. Stereotact Funct Neurosurg 2008;86:44-53.

17 Winkler D, Tittgemeyer M, Schwarz J, Preul C, Strecker K, Meixensberger J: The first evaluation of brain shift during functional neurosurgery by deformation field analysis. J Neurol Neurosurg Psychiatry 2005;76: 1161-1163.

18 Ganser KA, Dickhaus H, Metzner R, Wirtz CR: A deformable digital brain atlas system according to Talairach and Tournoux. Med Image Anal 2004;8:3-22.

19 Miocinovic S, Noecker AM, Maks CB, Butson CR, McIntyre CC: Cicerone: stereotactic neurophysiological recording and deep brain stimulation electrode placement software system. Acta Neurochir 2007;97 (suppl):561-567.

20 Guo T, Finnis KW, Parrent AG, Peters TM: Visualization and navigation system development and application for stereotactic deep-brain neurosurgeries. Comput Aided Surg 2006;11:231-239.

-21 Nowinski WL, Liu J, Thirunavuukarasuu A: Quantification and visualization of the three-dimensional inconsistency of the subthalamic nucleus in the SchaltenbrandWahren brain atlas. Stereotact Funct Neurosurg 2006;84:46-55.
22 Nowinski WL, Belov D, Pollak P, Benabid AL: Statistical analysis of 168 bilateral subthalamic nucleus implantations by means of the probabilistic functional atlas. Neurosurgery 2005;57:319-330.

23 Maks CB, Butson CR, Walter BL, Vitek JL, McIntyre CC: Deep brain stimulation activation volumes and their association with neurophysiological mapping and therapeutic outcomes. J Neurol Neurosurg Psychiatry, E-pub ahead of print.

24 Butson CR, Cooper SE, Henderson JM, McIntyre CC: Patient-specific analysis of the volume of tissue activated during deep brain stimulation. Neuroimage 2007;34:661-670.

25 Boyd S, Vanderberghe L: Convex Optimization. Cambridge, Cambridge University Press, 2004

26 Saint-Cyr JA, Hoque T, Pereira LC, Dostrovsky JO, Hutchison WD, Mikulis DJ, Abosch A, Sime E, Lang AE, Lozano AM: Localization of clinically effective stimulating electrodes in the human subthalamic nucleus on magnetic resonance imaging. J Neurosurg 2002;97:1152-1166.

27 Yelnik J, Damier P, Demeret S, Gervais D, Bardinet E, Bejjani BP, Francois C, Houeto JL, Arnule I, Dormont D, Galanaud D, Pidoux B, Cornu P, Agid Y: Localization of stimulating electrodes in patients with Parkinson disease by using a three-dimensional atlas-magnetic resonance imaging coregistration method. J Neurosurg 2003;99:8999.

28 Herzog J, Fietzek U, Hamel W, Morsnowski A, Steigerwald F, Schrader B, Weinert D, Pfister G, Muller D, Mehdorn HM, Deuschl G, Volkmann J: Most effective stimulation site in subthalamic deep brain stimulation for Parkinson's disease. Mov Disord 2004;19: 1050-1054.

29 Anheim M, Batir A, Fraix V, Silem M, Chabardès S, Seigneuret E, Krack P, Benabid $\mathrm{AL}$, Pollak P: Improvement in Parkinson disease by subthalamic nucleus stimulation based on electrode placement: effects of reimplantation. Arch Neurol 2008;65:612616.
30 Castro FJ, Pollo C, Meuli R, Maeder P, Cuisenaire O, Cuadra MB, Villemure JG, Thiran JP: A cross validation study of deep brain stimulation targeting: from experts to atlasbased, segmentation-based and automatic registration algorithms. IEEE Trans Med Imaging 2006;25:1440-1450.

- 31 Lemaire JJ, Coste J, Ouchchane L, Caire F, Nuti C, Derost P, Cristini V, Gabrillargues J, Hemm S, Durif F, Chazal J: Brain mapping in stereotactic surgery: a brief overview from the probabilistic targeting to the patientbased anatomic mapping. Neuroimage 2007; 37(suppl 1):S109-S115.

32 Guo T, Parrent A, Peters T: Surgical targeting accuracy analysis of six methods for subthalamic nucleus deep brain stimulation. Comput Aided Surg 2007;12:325-334.

33 Vitek JL, Bakay RA, Hashimoto T, Kaneoke Y, Mewes K, Zhang JY, Rye D, Starr P, Baron M, Turner R, DeLong MR: Microelectrodeguided pallidotomy: technical approach and its application in medically intractable Parkinson's disease. J Neurosurg 1998;88:10271043.

34 Acar F, Miller JP, Berk MC, Anderson G, Burchiel KJ: Safety of anterior commissureposterior commissure-based target calculation of the subthalamic nucleus in functional stereotactic procedures. Stereotact Funct Neurosurg 2007;85:287-291.

- 35 Xue Z, Shen D, Karacali B, Stern J, Rottenberg D, Davatzikos C: Simulating deformations of MR brain images for validation of atlas-based segmentation and registration algorithms. Neuroimage 2006;33:855-866.

36 Fletcher R: Practical Methods of Optimization. Chichester, Wiley, 1987.

37 Gill PR, Murray W, Wright MH: The Levenberg-Marquardt method; in Gill PR, Murray W, Wright MH: Practical Optimization. London, Academic Press, 1981, pp 136-137.

38 Hock W, Schittkowski K: A comparative performance evaluation of 27 nonlinear programming codes. Computing 1983;30:335.

39 Powell MJD: Variable metric methods for constrained optimization; in Bachem A, Grotschel M, Korte B (eds): Mathematical Programming: The State of the Art. Bonn, Springer, 1983, pp 288-311.

40 Collobert R, Bengio S: A gentle hessian for efficient gradient descent. Proc IEEE Int Conf on Acoust Speech Signal Processing, 2004, Montreal, vol 5, pp 517-520. 